\title{
Exploration and Research on the Category Management for the Chain Retail Enterprises: Taking Jiajiayue as Example
}

\author{
Shan Zhao \\ Weihai Vocational College, \\ Weihai , Shandong 264200 China
}

\begin{abstract}
In this paper, we conduct research on the category management for chain retail enterprises while taking Jiajiayue as the example. Category management theory research has more than ten years history, its core has been basically mature theory. The future study of category management focuses more on how to make the category management theory and be combined closely with the enterprise actual and pays more attention to the effect of the implementation and effect. Implementing category management investment return period is how to curve and how to evaluate the effect of the category management. The supermarket of the category management innovation is a huge project without a reasonable and predictable return that will be conducive to category management decision-making is unfavorable to the supermarket profits change after the implementation of category management right evaluation. Under this background, we propose our novel perspective on the corresponding issues to form the better theoretical analysis on the issues that is meaningful.
\end{abstract}

Keywords- Exploration, Category Management, Chain Retail Enterprises, Jiajiayue, Example.

\section{Introduction}

Chain platform of the operation management system, we can simply comb into three parts: operation management, training system, the supervision system. Operations management is the need to copy the content of the training system is the method of copying and the steering system is the guarantee of the supervision and control. It seems that chain enterprise training system construction that is a must to complete the task which is the bridge of the chain platform [1-2].
The efficiency of logistics distribution, to a large extent reflects and decides whole chain enterprise management level and management efficiency. In order to achieve the logistics of speed, good service and low cost of the basic goals, we must establish a reasonable and efficient distribution system that can be reflected from the listed aspects. (1) The rapidness is refers to the logistics operation speed, including the stock operation rapidness, sorting and distribution operation rapidness, rapidness and unloading the rapidness and service, etc. The rapidness of the logistics distribution system internal operation aimed at shortening the time of order processing and distribution main operation time, it is not only a link to speed up and it is to speed up the basic logistics distribution system. (2) Shipping information and transportation, warehousing and other links have close relations, plays a central role in logistics activities. To strengthen the management of shipping information to make the logistics distribution system as an organic whole, rather than the each link isolated activity. (3) Distribution operation standardization is according to requirements of modern logistics, the logistics distribution process and the specific distribution assignment to the specification, and establishing operation inspection and evaluation standards, in accordance with this standard specific operation organization and management, to improve the working quality of logistics distribution to reduce distribution costs.

The commodity space management in the position in the whole category management tactics is crucial, category management is in store level the ground. Space determines the category management tactics in the store location and commodity's position in the category, at the same 
time can also be for the space utilization efficiency to make the reasonable measure. Space management scope of work includes: the store each category display position, correlation, spatial allocation of the resources in the each category, category display principles, the unity and difference of basic different shops in space management, space efficiency analysis, etc. From the enterprise perspective, the space management can optimize space resource utilization efficiency to make more reasonable inventory level reduce the out of stock and high inventory and product unsalable phenomenon, so as to promote sales [3].

Enterprise management mode from the specific management concept, and on the specific goals, organizational resources, information and basic knowledge, conduct operational activities of the basic framework, rules and methods as summarized from the dimension of specific management concept and management methods, with different scholars summed up by the enterprise management mode is necessary always, different types, dynamic, and always, but if summed up from the dimension of the management goal and basic management idea, different enterprise management mode in its essence is consistent with the actual. The figure one demonstrates the corresponding principles.

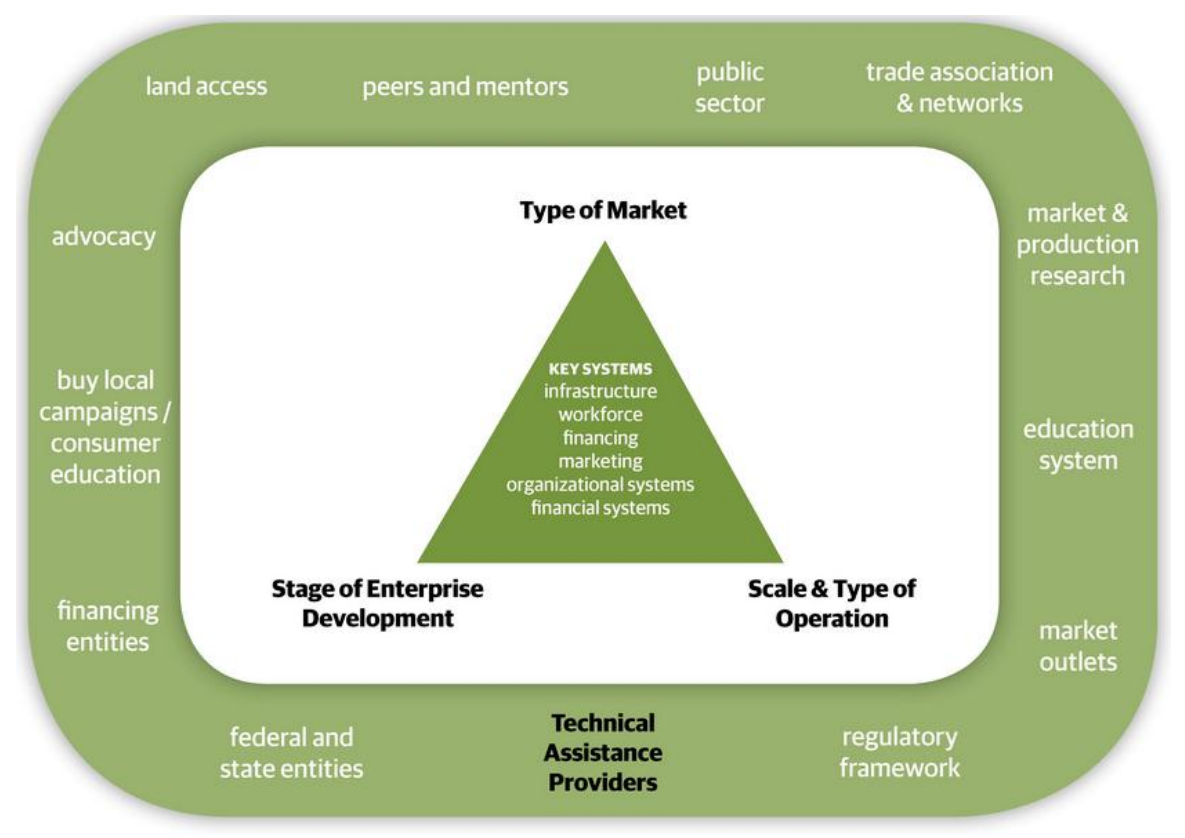

Figure 1. The General Components of the Chain Retail Enterprise Management

In this paper, we conduct research on the category management for the chain retail enterprises while taking Jiajiayue as the example. In the modern enterprise management to clear responsibilities and division of labor, namely general management as managers need to do is to macro control, to the end rather than the process, and specific handle basic affairs personnel pay attention to the process with everyone to do their jobs seriously, send together implement to achieve management objectives. In the later sections, we will discuss the related issues in detail.

\section{The Proposed Methodology}

The Characteristics of the Jiajiayue. Supermarket chain, the core competitiveness, in large part of the upper management ability on the core product, at present China's supermarket industry peers are identified with the fresh food is the core of supermarket products that is the 
earliest start in Chinese supermarket stores the supermarket for fresh food comprehensive proprietary company, and become in the management level of fresh food one of the country's leading supermarket company [4-5].

Based on such consideration, the terminal supermarket chain management of fresh agricultural products supply chain management model can be directly or through agricultural association, brokers, centralized purchasing raw agricultural production base, the decrease of the intermediate links, to ensure product freshness and safety, to establish effective circulation channel directly, can really producers and consumers interest. At the same time, with the improvement of residents' consumption level in China and to the improvement of fresh agricultural products, through a supermarket to buy fresh, safety of fresh agricultural products become the choice of many residents.

Build based on the pattern of supermarket chain of the fresh agricultural products supply chain management conditions for Jiajiayue could be summarized as follows. (1) Supermarket chains should have more abundant capital strength, the brand assets and channel conditions. Supermarket chain to become the core of the supply chain enterprise must have the funds, brand, information, channels, the advantages of the reputation, to attract other node enterprises of supply chain to join to the dominant in the supply chain, to have control of the whole supply chain operation and management, the upper reaches of the network of suppliers, manufacturers, distributors, etc. to be in a subordinate position, assume their respective responsibilities, must collaborate together to try to meet needs of consumers. (2) Supermarket chain to be more perfect logistics distribution system. In order to produce the rapid response to changes in consumer demand, improve the general quality and safety of fresh agricultural products, to provide the consumers with the safe, fresh, diversified and innovative purposes of fresh agricultural products, large chain supermarket must have its own distribution system [6].
The Category Management. Category management six main factors include strategy, business process, rating scale, ability of organization, information technology and trading partners relations of cooperation, there are two key elements is strategies and business processes. In order to make the core essential factor effectively, the other four to this element, namely the general rating scale, ability of organization, information technology and trading partners relations of cooperation that is the six key elements in performing category management decision-making and critical business processes.

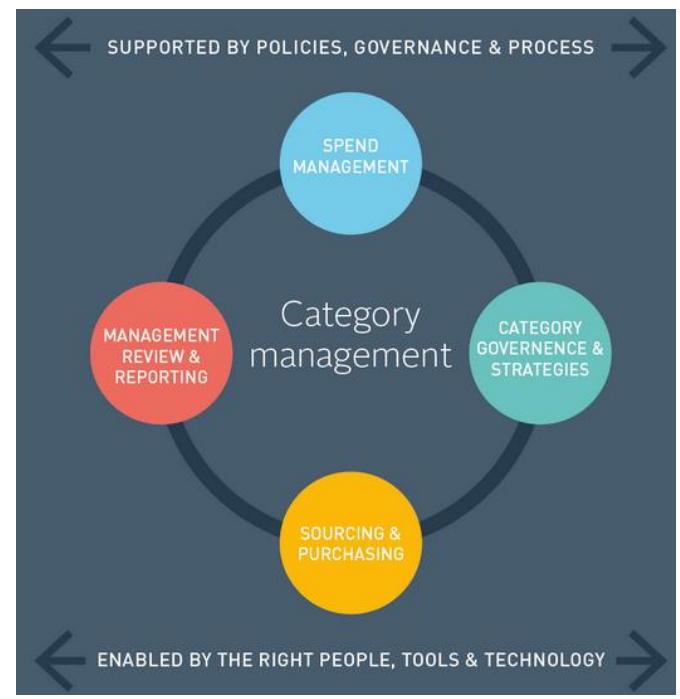

Figure 2. The Primary Architecture of the Category Management

The entire retail industry to buy hand-made and platform is the ultimate goal, the goal may be under the drive of electronic commerce, as will arrive ahead of time, ten years or twenty years, as also depends on the industry of capital concentration and speed of the transformation of Chinese consumer society. But before the industry transition, back in the nature of retail has several important milestones on the road, each go to a milestone, is in a step toward the ultimate goal at a lower cost, progressive training talents, cultivate the understanding of customers, develop sensitivity to fashion as deepen the relationship with basic suppliers. Don't do subversive 
revolution, need to gradual improvement, each milestone is an effective improvement.

Many varieties of the brands, although signed the strategic cooperation agreement, but due to the varieties of brand cooperation benefits only leave chain headquarters, products in stores because of the high gross margin and failed to make the inspection variety range, to cooperate with sales force is difficult to reflect. Chain if mainly sales assessment item, then we can set general strategic partners, cooperation brand products to assess the varieties, stores and the assistant will not deviate from the headquarters strategic orientation, the brand enterprise strategic cooperation execution brand products can smoothly through the strategic cooperation force [7-8].

The Chain Enterprise Features. For chain enterprises, the importance of logistics distribution is: can speed up the circulation of commodities, save the circulation time, promote the virtuous cycle of the commodity production that can be generally reflected from the listed aspects.

- Brand can say is the life of the chain, it is the guarantee of the chain enterprise services and quality. Chain enterprises are able to obtain the sustained and the rapid development, to some extent is also chain brand effect in the process of amplification.

- In the chain enterprises, standardization is the guarantee enterprise on the basic principle of the low cost operation, is a distribution center and purchasing department efficient large-scale operation of the premise, with the strategic goals of fraught with change is closely related.

- The highest state of culture chain is a chain. Due to the chain operation is not quite as strict as general enterprise subordinate relations. Therefore, the headquarters for the joining trader implement effective management difficulty is big. This time, except on general standardized management, we must also have a cultural management.
Convenience store chain's core business processes. Core process refers to the process to create value for the customer the product or service activities. According to the characteristics of the chain convenience store formats, convenience stores to provide customers with value is mainly a reasonable price, shopping convenience, comfort and pleasure of buying process and the new products constantly updated. In traditional convenient chain enterprise, due to the division of labor between departments, the process is often artificially divided, each department is responsible for the process on a only, no one is responsible for the whole process, therefore in the process of reengineering should ensure the integrity of the process as much as possible. Traditional convenience store chain market research consciousness is not strong, don't even have a clear market research process, due to new product development and stores the actual demand from consumers, usually restricted the ability of enterprise management, through the process to design, market research and new product development processes and effective integration, thus improve effectiveness of product development and promotion plans.

The Future of the Business Management Pattern. Humanistic management in the enterprise human resources management is a kind of strict self-discipline of the scientific human management methods. Enterprises to promote cultural and people-oriented humanized management will in turn reflects the three aspects of the evolution process of the transformation of the human relations, the concrete is the relationship between the managers and by managers of change, from the subordinate relations gradually become a partnership as the value of employees themselves becomes a part of the enterprise value. Enterprise culture to enterprise staff's thought, the psychological and the behavioral constraints and norms and the constraint function of enterprise culture is mainly done by improve the system of management and general overall ethics that can be reflected from the listed aspects. 
To better implement this principle requires managers to make all employees to be satisfied as it is also a basic requirement and general target of the humanistic management. In financial management, not only to guarantee the interests of the top leaders and technical elite that also has to meet the middle management and the interests of the employees.

Law of emotion is a kind of the emotional management rule. Require financial managers in enterprises in the management of the specific to learn to solve problems related to the use of the emotional factors. To make every employee to learn the perspective-taking problem, this also is the common way of the management in humanistic management doctrine, refused to blindly resistance or blindly obedient, and reflect on yourself, have a problem if you have a problem is to start from their own, if you are right that should be advocated their positions and not because of superior ideas and changed.

Balance law, refers to the actual financial enterprise management to achieve people-oriented is the need to man and his environment balance each other. This is humanistic management idea in the management of financial firms a scientific law, by focusing on enterprise ecosystem of people as fundamental management concept. Because of the individual factors of ecological system in there is out of sync between mine and the relationship of one honor.

\section{Conclusion}

In this paper, we conduct research on the category management for the chain retail enterprises while taking Jiajiayue as the example. Chain was born in relatively developed commodity economy stage, at this point, the purchasing power of consumers has reached a certain level purchasing psychology mature, diversified consumption pattern has been formed. As a result, target marketing is the most chain enterprise should adopt strategy. In this way, the chain will be targeted and specific goals for their marketing activities. Category management and supplier's ultimate purpose is to try to retail partner strength successfully combined with the implementation to meet consumer demand as the guide, create customer value for the purpose of the category management business process, in order to obtain the growth of the companies on the performance and the overall benefit. In the recent future, we will conduct more theoretical research for enhancing the current methodology.

\section{Acknowledgement}

This paper is supported by the Weihai vocational college department of economic management. The project name is: exploration and research on the category management for the chain retail enterprises: taking Jiajiayue as the example (NO. 2014KY020).

\section{Reference}

[1] Aihua, S. H. A. O. "Job Stressors and Management Tactics of Female Employees in Chain Retail Business." Organization 87 (2013): 1.

[2] Ahlert, Dieter, and Benjamin Schefer. "Management Summary: The Liberation of the Value Chain from Cartel-Law Restraints'." Vertical Price Coordination and Brand Care. Springer Berlin Heidelberg, 2013. 59-70.

[3] Ivanov, Gennady, and Elena Mayorova. "Intangible assets and competitive advantage in retail: case study from Russia." Asian Social Science 11.12 (2015): 38.

[4] Min, Hai Qiang, and Chen Xu. "Industrial synergy mechanism and operation mode research based on the retail industry." Applied Mechanics and Materials. Vol. 380. 2013. 
[5] Shin, Seungjae, and Burak Eksioglu. "An empirical study of RFID productivity in the US retail supply chain." International Journal of Production Economics 163 (2015): 89-96.

[6] Hsiao, Lu, and Ying - Ju Chen. "Strategic motive for introducing internet channels in a supply chain." Production and Operations Management 23.1 (2014): 36-47.

[7] Minten, Bart, K. A. S. Murshid, and Thomas Reardon. "Food quality changes and implications: Evidence from the rice value chain of Bangladesh." World Development 42 (2013): 100-113.

[8] Liu, Bin, et al. "Research on the supply chain risk evaluation of manufacturing enterprises." 2012 International Conference on Computer Science and Electronics Engineering. IEEE, 2012. 